\title{
Fine-Needle Aspiration Cytology: An Advancing Horizon
}

\author{
Darshana Jhala, ${ }^{1,2}$ Aileen Wee, ${ }^{3,4}$ Gary Tse, ${ }^{5}$ and Zubair Baloch ${ }^{1}$ \\ ${ }^{1}$ Department of Pathology and Laboratory Medicine, University of Pennsylvania, Philadelphia, PA 19104, USA \\ ${ }^{2}$ Pathology and Laboratory Medicine, Philadelphia VA Medical Center, Philadelphia, PA 19104, USA \\ ${ }^{3}$ Department of Pathology, Yong Loo Lin School of Medicine, National University of Singapore, \\ 21 Lower Kent Ridge Road, Singapore 119077 \\ ${ }^{4}$ Department of Pathology, National University Hospital, 5 Lower Kent Ridge Road, Main Building, Level 3, Singapore 119074 \\ ${ }^{5}$ The Chinese University of Hong Kong, Shatin, Hong Kong SAR, Hong Kong \\ Correspondence should be addressed to Darshana Jhala, darshana.jhala@uphs.upenn.edu
}

Received 24 August 2011; Accepted 24 August 2011

Copyright ( 2011 Darshana Jhala et al. This is an open access article distributed under the Creative Commons Attribution License, which permits unrestricted use, distribution, and reproduction in any medium, provided the original work is properly cited.

The current practice of "Fine Needle Aspiration Cytology (FNAC) has established itself as an important modality in the diagnosis and management of superficial and deep seated lesions throughout the body. With this wide employment of FNAC, the cytopathologists play a pivotal role in the management and therapeutic decisions that are based on the diagnosis of these limited FNAC samples. Furthermore, the use of FNAC samples for biomarker research is advancing rapidly and is being widely investigated and applied for the treatment and prognostic purposes.

In this special issue, we have made an attempt to provide a "flavor" of the current practice of FNAC with an emphasis on correlation of tissue biopsies with PET results, FNAC of breast, liver, pulmonary, and head and neck lesions. Similarly, the use of FNAC in the diagnosis of Langerhans cell histiocytosis, neuritic leprosy, and granulomatous inflammation is also discussed.

With the increasing use of image-assisted FNAC, pathologists are now an integral part in the diagnosis and management of the deep seated lesions. In lieu of this, it has become important for the pathologists to be aware of the limitations of different imaging techniques. The paper on the correlation of tissue biopsies with PET results discusses the limitations of an increased SUV value on PET scan.

The use of fine needle aspiration cytology has been proven to give fast, economical, and valuable diagnosis of palpable breast lumps. In this issue, the role of FNAC in the evaluation of breast lump in a high patient volume center is addressed with emphasis on the importance of skill and training for both pathologists and technicians to prevent suboptimal sampling, thus, increasing the reliability of the procedure. Another important but not commonly discussed aspect of breast FNAC - the nonmalignant categories-is also addressed, with a review of the cytomorphology of benign breast lumps, some of which could be mistaken for malignancy due to the diaphanous appearance and overlapping cytologic features. The false negative and false positive FNAC is further discussed in detail so as to avert misinterpretation. These provide practical information for readers when dealing with FNAC of breast lesions.

The paper on liver FNAC covers various aspects and discusses the role of FNAC in liver lesions. There is an active debate about the preoperative/pretransplantation diagnostic role of FNAC of hepatocellular carcinoma (HCC) and precursor lesions, especially in the face of advances in dynamic imaging techniques. New trends in personalized molecular targeted therapy require better characterization and prediction of HCC behavior. FNAC biopsy technique is still the most minimally invasive approach for the procurement of tumor and peritumoral tissue for molecular studies. Thus, in the near future, hepatic FNAC is likely to become a point of care in the management of HCC patients, especially inoperable cases.

In the current era of personalized medicine, the FNAC diagnosis of nonsmall cell carcinoma for a pulmonary nodule is no longer considered an adequate diagnosis. Pathologists are often required to further subclassify these in to adenocarcinoma and squamous cell carcinoma. With the increasing use of image-assisted FNAC including endobronchial 
ultrasound guided FNAC (EBUS-FNA), cytologic or small biopsy material has become the only form of tissue available for diagnosis. The paper on FNAC of pulmonary lesions reviews the current concepts in the suitability and accuracy of FNAC in lung cancers including diagnosis, classification, use of ancillary techniques, and prognostic marker assessment.

FNAC is a valuable technique in the workup of nodules and masses arising within the head and neck region. It is primarily utilized to confirm or exclude the diagnosis of malignancy involving head and neck organs especially lymph nodes, thyroid, and salivary glands. It has been shown that FNAC of salivary gland lesions is a valuable way to preoperatively assess lesional tissue, determine the need for surgical intervention, and assist in planning the appropriate surgical approach prior to resection. In this issue, the manuscript on cytologic diagnosis of mucoepidermoid carcinoma discusses the role of FNAC in the diagnosis of mucoepidermoid carcinoma (MEC), the common malignant tumor affecting parotid gland. In addition, it also brings forth how the rare and recently described oncocytic variant can pose problems in the diagnosis of MEC.

In conclusion, this special issue includes a potpourri of topics which provides a thoughtful glimpse into various techniques, diagnostic ability, and limitations of the current practice of FNAC.

Darshana Jhala Aileen Wee Gary Tse Zubair Baloch 


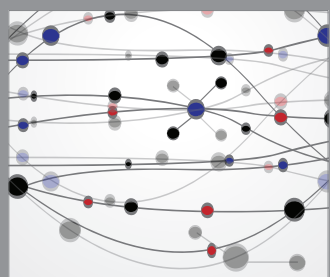

The Scientific World Journal
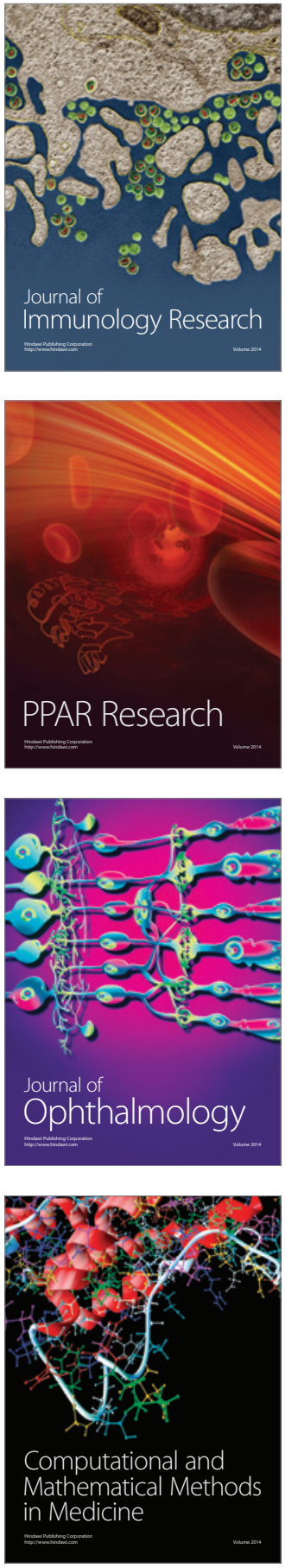

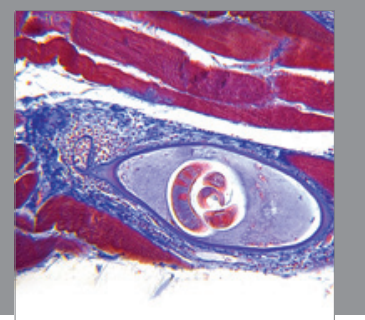

Gastroenterology

Research and Practice
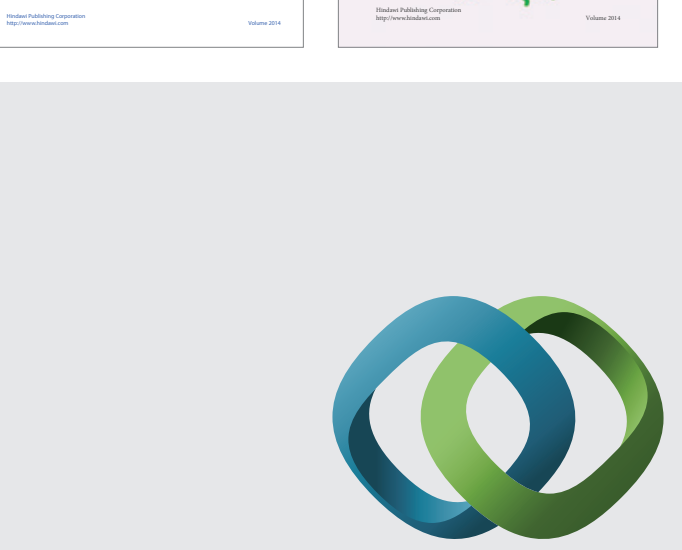

\section{Hindawi}

Submit your manuscripts at

http://www.hindawi.com
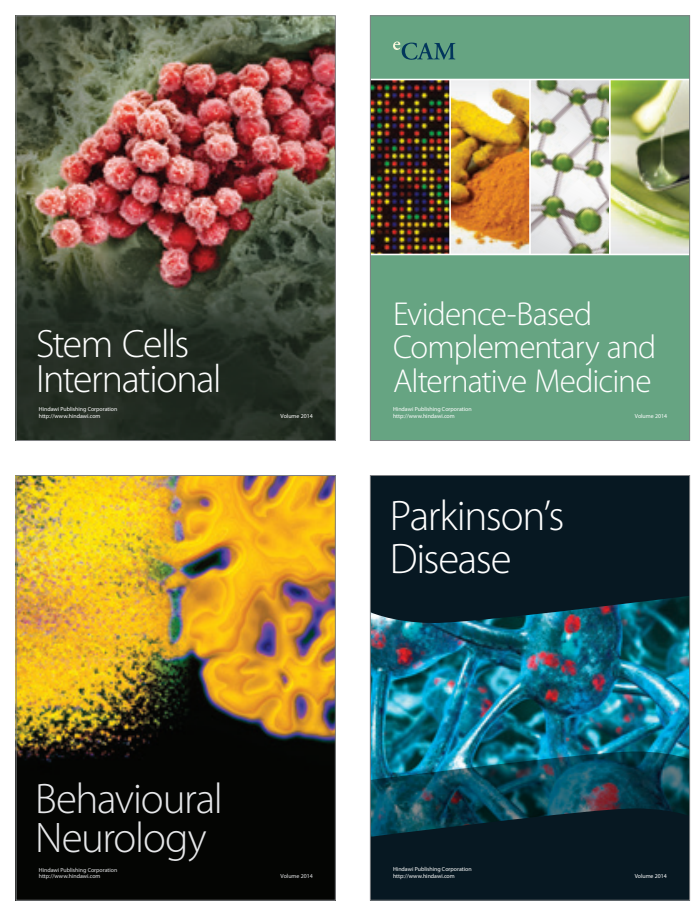

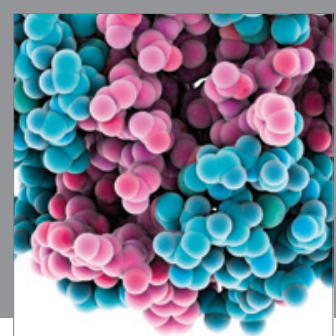

Journal of
Diabetes Research

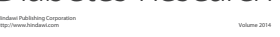

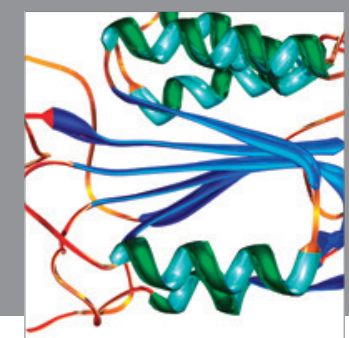

Disease Markers
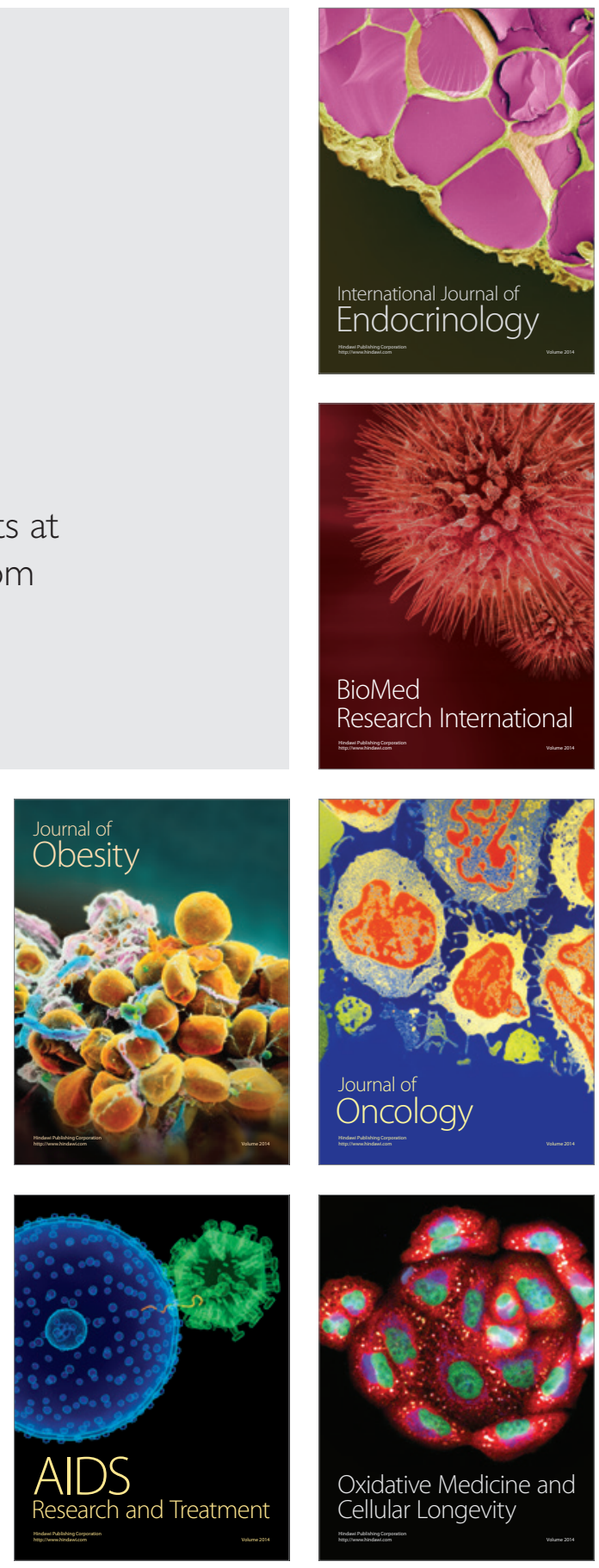